\title{
Breast self examination and breast cancer: Knowledge and practice among female medical students in a Kenyan university
}

\author{
Kimani S.M., BSc (Hons), Fourth year medical student and Muthumbi E., Third year medical student, \\ School of Medicine, University of Nairobi, Kenya \\ Corresponding author: Kimani S.M., Department of Human Anatomy, University of Nairobi, \\ P.O. Box 30197-00100, Nairobi, Kenya, Email: mburukim2003@yahoo.com
}

\section{Abstract}

BACKGROUND: Medical students play an important role in creating a supportive environment within their communities for screening behaviours in health promotion. Medical students must possess the appropriate knowledge concerning breast self examination (BSE) and breast cancer to be effective health educators.

OBJECTIVE: To investigate the level of knowledge concerning BSE and breast cancer and to evaluate the practice of BSE among female medical students.

MATERIALS AND METHODS: A crosssectional descriptive study conducted between March and June 2008 involving female students drawn from schools within the College of Health sciences, University of Nairobi. The questionnaires contained items on the biodata, knowledge and practice of BSE and knowledge on breast cancer. Responses on awareness on breast cancer were weighed using a 3-point Likert's scale.

RESULTS: A total of 169 respondents were interviewed. Majority (94.6\%) had heard about BSE with $114(69.9 \%)$ having ever practiced it. Less than $20 \%$ of the respondents performed BSE regularly. There were no significant differences between clinical and preclinical students. Mass media was the most common source of information (45.2\%). Relatives and formal lectures formed the least $(7 \%)$ source of information for the respondents. Significant interschool differences on knowledge and practice are presented.

CONCLUSION: Despite being aware, a significant proportion of female medical students do not practice BSE. Information dissemination regarding breast health is underprovided among family members.

RECOMMENDATION: Programs targeting improved propagation of information and BSE especially among medical trainees should be instituted so as to strengthen health promotion in the community.

KEY WORDS: Breast self examination, breast cancer, female medical students

\section{Introduction}

In the last few years, the rising incidence of breast cancer and increasing mortality from the disease is emerging as major health concerns in developing countries (1). The lifetime risk of a woman getting affected by breast cancer is up to 1 in 10 (2). The primary reason for the escalating mortality is late diagnosis of the disease and a lack of early detection program $(3,4)$. Early 
detection through screening ensures maximum benefit is acquired from the available treatment options (3).

The current guidelines from the American Cancer Society currently advocates for monthly breast self examination (BSE) among women 20 years and older (5). Other early detection techniques include clinical breast examination, ultrasonography and mammography (6). Unlike the latter methods, BSE is a simple, cheap, noninvasive and readily available method for the detection of early breast cancer in women (2). There is evidence that women who correctly practice BSE monthly are more likely to detect a lump in the early stage of its development (7).

There exist conflicting views on the value of BSE in breast cancer management $(8,9)$. Descriptive, case-control and cohort studies on the effectiveness of BSE in reducing mortality in breast cancer have yielded contradictory results (10). Extensive randomized controlled trials under the Cochrane database review in Russia and China have shown no significant effect of regular BSE on breast cancer mortality or incidence (11). Despite obvious study design flaws, these trials involved developed nations where dynamics of healthcare are different from those of developing nations. The Canadian National Breast Screening Study (NBSS) point to the need for proper education in breast self-examination as an important component of the screening program (12). In the UK Trial of Early Detection of Breast Cancer, yearly self examination resulted in a significant decrease in breast cancer (13).

Health workers play an important role in creating an environment supportive of screening behaviours by offering positive role models, reinforcing social norms favourable toward screening and helping to develop supportive institutional policies (14-16). In order to be effective in their role as health educators, health care workers must possess the appropriate knowledge, attitude and beliefs concerning the health behaviour being promoted (17). Medical students, who are an integral part of the health provision system, present a multiple facet of the community due to the diverse backgrounds. As such, they represent an invaluable resource in encouraging screening behaviours in health promotion. The present study assessed knowledge and practice of breast self-examination among female medical students in a Kenyan University.

\section{Materials and Methods}

Subjects: The study was conducted between March and June 2008. Respondents were drawn from the schools of medicine, pharmacy, dental sciences and nursing sciences at the College of Health Sciences, University of Nairobi. Enrolment was on a voluntary basis.

Data collection: A self-administering structured questionnaire was developed, pretested and used to collect data. The questionnaire was administered only once with an overall response rate of $40 \%$. Demographic characteristics captured included age, marital status, level and course of study and religion. The tool collected the respondents' knowledge on breast cancer as well as on awareness, source of information, and practice of BSE. Responses were weighed using a 3-point Likert's scale. In addition, the knowledge and practice of BSE was compared between the preclinical (Level two) and clinical years (Level three, four and five) using Chi square.

Data analysis: The data was entered and analyzed using SPSS 16.0 statistical software (SPSS Inc., Chicago, Illinois). Frequencies, means and standard deviations were calculated. A p-value $<0.05$ was considered significant.

\section{Results}

Demographics: A total of 169 respondents fully completed the questionnaires. The age of the respondents ranged from 19 to 25 (mean=21 years). Other demographic characteristics are shown in Table 1.

Breast self examination awareness and practice: A majority of respondents $(94.6 \%)$ had heard about BSE, the mass media (radio, magazines and television documentaries) being the commonest $(45.2 \%)$ source of information. Information from 
Table 1: Characteristics of women surveyed concerning breast self-examination and breast cancer

\begin{tabular}{llcc}
\hline Variable & Categories & No. & (\%) \\
\hline Religion & Christian & 139 & 82.2 \\
& Muslim & 17 & 10.1 \\
& Hindu & 3 & 1.7 \\
& Others e.g. African sects, Sikhism & 10 & 5.9 \\
Faculty enrolled & Medicine & 92 & 54.4 \\
& Pharmacy & 41 & 24.3 \\
& Dental sciences & 16 & 9.5 \\
& Nursing & 20 & 11.8 \\
Marital status & Single & 164 & 97.0 \\
& Married & 5 & 3.0 \\
& Second & 85 & 50.3 \\
& Third & 35 & 20.7 \\
& Fourth and fifth & 49 & 29.0 \\
\hline
\end{tabular}

Figure 1: Source of information on breast self-examination

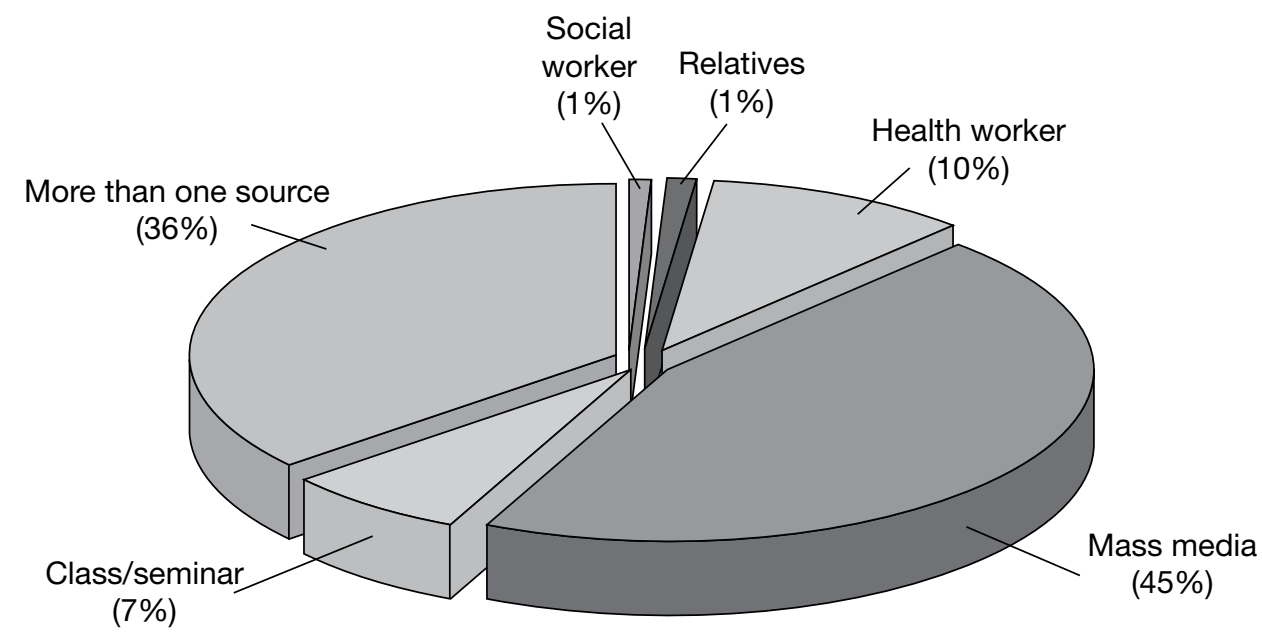

relatives and other social networks was scarce (Figure 1). The prevalence of self-reported BSE was $69 \%$ with about a fifth of the respondents performing BSE monthly. Less than $4 \%$ of the respondents had been exposed to other screening methods such as ultrasonography. The level of awareness and practice of BSE did not show significant differences between the preclinical and clinical years (Table 2).

Breast cancer-related information: Twenty three (13.8\%) of the respondents had a positive family history of breast cancer. Aunts and distant relatives were the most commonly (86.9\%) affected relatives. Symptoms of breast disease were reported by $53(32.9 \%)$ respondents and included; breast pain (29.4\%), lump (13.1\%), nipple discharge $(2.5 \%)$ and skin colour changes $(1.9 \%)$.

An analysis of other risk factors for breast cancer revealed that there was inadequate knowledge on the relationship between breast feeding and breast cancer with up to $65 \%$ of the respondents in the present study not being able to correctly identify the appropriate response on the Likert's scale. 
Table 2: Likert's scale scores on breast cancer-related knowledge levels

\begin{tabular}{|c|c|c|c|c|c|}
\hline \multirow[t]{2}{*}{ Variable } & \multicolumn{2}{|c|}{ Preclinical } & \multicolumn{2}{|c|}{ Clinical } & \multirow[t]{2}{*}{$P$ value } \\
\hline & No. & $(\%)$ & No. & $(\%)$ & \\
\hline Number of students who had heard about BSE & $82 / 85$ & 96.5 & $78 / 84$ & 92.9 & 0.810 \\
\hline Number of students who practice BSE & $58 / 85$ & 68.2 & $35 / 48$ & 72.9 & 0.287 \\
\hline
\end{tabular}

Table 2: Likert's scale scores on breast cancer-related knowledge levels

\begin{tabular}{lccc}
\hline Question & Yes (\%) & Not sure (\%) & No (\%) \\
\hline Family history of breast cancer may increase one's chances of getting breast & 90.6 & 6.2 & 3.1 \\
cancer & 76.4 & 20.5 & 3.1 \\
The incidence of breast cancer in Kenya is increasing & 34.4 & 59.4 & 6.2 \\
Women who breastfeed have reduced risks of breast cancer & 52.5 & 32.5 & 15 \\
Breast cancer is more common in elderly than teenage women & 94.4 & 3.8 & 1.9 \\
Early detection of breast cancer may improve treatment & 55.6 & 38.1 & 6.2 \\
High fat diet increase the risk of breast cancer & 50 & 41 & 9 \\
Routine alcohol consumption increases the risk of breast cancer & 12 & 34.2 & 53.8 \\
Tobacco smoking has minimal effect on breast cancer risk & 90.5 & 1.9 & 7.6 \\
Breast self examination may help one detect breast disease & 4.4 & 7.5 & 88.1 \\
Breast cancer always kills & 3.8 & 1.3 & 95 \\
Breast self examination should be initiated to married women alone & 12.6 & 40.3 & 47.2 \\
Mammography is very painful and uncomfortable & 48.7 & 45.6 & 5.7 \\
Mammography is capable of detecting small neoplasm & &
\end{tabular}

There were significant differences noted between respondents in the different schools for the role of breast feeding in breast cancer $(p=0.028)$. Majority of students in the faculty of medicine positively identified that breast feeding reduces the risk while most students in the faculty of pharmacy $(62.5 \%)$, dental sciences $(86.7 \%)$ and nursing sciences $(70.0 \%)$ were not sure of the association between breast feeding and breast cancer.

\section{Discussion}

The present study shows that a majority of respondents $(94.6 \%)$ had heard about BSE. Even though similar studies among medical students are scarce, awareness on BSE has been reported to be $16.4 \%$ in a rural Indian population (18). These differences could be attributed to the older age and rural location of the latter population. Despite the high level of awareness, only two- thirds of the respondents had ever practised BSE in the population studied. This compares favourably with a study among Jordanian nurses where more than $52 \%$ reported practising BSE (2). Lower prevalence of self-reported BSE has also been noted in other populations $(1,20)$.

The American Cancer Society recommends that women above the age of 20 years should perform monthly BSE. Pertinent to this, are observations from the present study that only $19.2 \%$ of the respondents reported performing monthly BSE. Similar studies have reported slightly higher prevalence of self-reported BSE ranging from $30-50 \%(1,2,17,19)$. The young age of the respondents in the current study may have contributed to the difference. On the other hand, considering that the respondents in the present study were drawn from the health profession, discrepancy between knowledge and practice is unacceptable. Future breast health programs should seek to address this disconnect. 
In a study among women in Trinidad and Tobago, it was found that television, newspaper and radio formed the major source of information on BSE by over $70 \%$ of the respondents (1). The findings are similar to our study but with a lower figure of $45.2 \%$. This differences could be explained by the slight variation in the population characteristics between the two studies; the Caribbean study enrolled slightly older women (mean age = $40)$. In the present study, health workers were cited as a source of information by $10 \%$ of the respondents. In contrast, the Caribbean study reported that more than $44 \%$ of the respondents obtained the information on BSE from a health worker. This difference between the two populations can be attributed to the fact that health workers in the Caribbean country are usually trained on offering BSE education and promotion.

In conclusion, despite being aware, a significant proportion of female medical students do not practice BSE. Information dissemination regarding breast health is underprovided among family members.

\section{Recommendation}

A community oriented educational intervention program emphasizing on importance of early detection in the management of breast cancer can bring about the desirable change among women.

\section{Limitations}

Study bias was possible since the population assessed represented medical trainees who are likely to be more informed on health issues than the general public. Furthermore, this study sought to investigate common beliefs of the participants; therefore the possibility of having missed other beliefs within the population cannot be ruled out.

\section{Acknowledgements}

We would like to acknowledge all the respondents who took time to complete the questionnaires.

\section{References}

1. Modeste N.N., Caleb-Drayton V.L. and Montgomery S. Barriers to early detection of breast cancer among women in a Caribbean population. Pan. Amer. J. Public Health. 1999; 5(3): 152-156.

2. Ali Abu-Salem O.T. and Abdulla Hassan M. Breast self-examination among female nurses in Jordan. Shiraz E-Med. J. 2007; 8(2): 51-57.

3. Parkin D.M. Cancer in developing countries. Cancer Surv. 1994; 19-20: 519-561.

4. Pinotti J.A., Barros A.C., Hegg R., et al. Breast cancer control program in developing countries. Breast Dis. 1995; 8: 243-250.

5. American Cancer Society. Breast self-examination: a new approach [pamphlet] [Report]. - Unknown, California: ACS, 1996. Publication 6438.39

6. Jatoi I. Screening clinical breast examinations. Surg. Clin. North Amer. 2003; 83(4): 789-801.

7. American Cancer Society. Cancer Statistics. Amer. Cancer J. Clin. 2002; 52: 10-11.

8. Gehrke A.W. Breast self-examination: A mixed message. J. National Cancer Institute. 2000; 92: 11201121.

9. Larkin M. Breast self-examination does more harm than good, says task force. Lancet. 2001; 357: 2109.

10. Hislop T.G. Is breast self-examination still necessary? Can. Med. Assoc. J. 1997; 157: 1225-1226.

11. WillettL.Limited evidenceon regular breastexamination does not support its effectiveness for reducing breast cancer deaths. Evid. Based Med. 2003; 8: 169.

12. Morrison B.J. Screening for breast cancer. In: Canadian Task Force on the Periodic Health Examination. The Canadian guide to clinical preventive healthcare. Ottawa: Health Canada; 1994. p. 788-95. Cat no H212117/1994E.

13. 16-year mortality from breast cancer in the UK Trial of Early Detection of Breast Cancer. Lancet. 1999; 353: 1909-1914. 
14. Clarke D.E. and SandIer L.S. Factors involved in nurses' teaching breast self-examination. Cancer Nursing. 1989; 12: 41-46.

15. Fletcher S.W., O'Malley M.S., Earp J.L., et al. How best to teach women breast self-examination. A randomized controlled trial. Ann. Inter. Med. 1990; 112: $772-779$.

16. Bastani R., Marcus A.C. and Hollatz-Brown A. Screening mammography rates and barriers to use: a Los Angeles County survey. Prev. Med. 1991; 20: 350-363

17. Bastani R., Maxwell A.E., Carbonari J., et al. Breast cancer knowledge, attitudes, and behaviors: a comparison of rural health and non-health workers. Cancer Epidemiol. Biomarkers \& Prev. 1994; 3: 77-85.

18. Rao R.S.P., Suma N., Nair N.S. and Kamath V.G. Acceptability and effectiveness of a breast health awareness program for rural women in India. Indian J. Med. Sci. 2005; 59(9): 396-402.

19. Budden L. Registered nurses' breast self-examination practice and teaching to female clients. J. Comm. Health Nur. 1998; 15: 101-112.

20. Haji-Mahmoodi M., Montazeri A., Jarvandi S., et al. Breast self-examination: Knowledge, attitudes, and practice among female health care in Tehran, Iran. Breast. 2002; 4: 222-225. 